\title{
A Smart Hip Abduction Splint For Developmental Dysplasia
}

\author{
Nada Ayoub ${ }^{1}$, Sarah Sobh ${ }^{1}$, Hussein Salah Eldeen ${ }^{1}$ and Amira J. Zaylaa ${ }^{1,2,3 *}$ \\ ${ }^{1}$ Department of Biomedical Engineering, Lebanese International University, Bekaa, Lebanon \\ ${ }^{2}$ Neoroscience Research Center, Faculty of Medical Sciences, Lebanese University, Beirut, Lebanon \\ ${ }^{3}$ Faculty of Public Health-V, Lebanese University, Lebanon \\ *Corresponding Author, amira.zaylaa@ul.edu.lb
}

\begin{abstract}
Developmental dysplasia of the hip is a special condition that faces newborn babies, it may happen due to genetic factors or during delivery. Pavlik harness was the solution, however, it caused suffering to babies due to the presence of straps that are very uncomfortable and cause pain and itching. Our aim is to develop a new smart hip abduction device which allows the physician's in charge of the baby to control automatically and accurately the degree of opening of the device, and to evaluate the performance of the smart device statistically. The automatic control was achieved by a touchscreen which controls the servomotors and the degree of treatment. Simulation results showed the compact design which could reduce the pain of the baby and help him/her recover with less suffering. Experimental results showcased the compact design of the Smart Hip Abduction Splint providing a comfortable and flexible movement of the baby. The device User Interface and touchscreen results showed that it allows the physician to set the angle of treatment accurately in less than a second and in a feasible way. Also, valuable instructions and information were provided on the devices' screen. Statistical evaluation showed that our smart abduction hip device surpasses the pavlik harness in its performance. Our Smart HAS provided $\mathbf{9 8 \%}$ of accuracy in treatment, $89 \%$ safety and $100 \%$ automatic control. For further improvement, various sensors that ensure the maximum safety and monitor the baby's treatment could be considered.
\end{abstract}

Index Terms-Smart Device; Hip Abduction Splint; Developmental Dysplasia; Communication; Biomedical Instrument; Statistical Evaluation.

\section{INTRODUCTION}

Babies' medical problems are dramatically increasing, such as Developmental Dysplasia of the Hip (DDH). DDH is a congenital (present at birth) condition of the hip joint. It occurs once in every 1,000 live births [1]. The hip joint is developed as a ball and socket joint. In DDH, the hip socket may be shallow, letting the "ball" of the long leg bone, also known as the femoral head, slip in and out of the socket. The ball may move partially or completely out of the hip socket [1]. The degree of hip looseness, or instability, varies among children with DDH. In the most severe cases of DDH, the head of the femur is completely out of the socket, i.e dislocated. Thereby, the head of the femur lies within the acetabulum, but can easily be pushed out of the socket during a physical examination. In mild cases of DDH, the head of the femur is simply loose in the socket, i.e. subluxable. During a physical examination, the bone can be moved within the socket, but not dislocated [2]. Hip dysplasia is considered a multifactorial trait. Multifactorial inheritance means that many factors are involved in causing a birth defect. The factors are usually both genetic and environmental. The greatest incidence of DDH occurs in first-born females (6:1) [1], with a history of a close relative with DDH. One of the environmental influences thought to contribute to hip dysplasia is the baby's response to the mother's hormones during pregnancy. A tight uterus that prevents fetal movement or a breech delivery may also cause hip dysplasia. The left hip is involved more frequently $(60 \%)$ than the right due to intrauterine positioning [1]. First-born babies are at higher risk since the uterus is small and there is limited room for the baby to move; therefore, affecting the development of the hip. There are other factors such as, family history of $\mathrm{DDH}$, very flexible ligaments, or the position of the baby in the uterus, especially with breech presentations or associations with other orthopedic problems that include metatarsus adductus, clubfoot deformity, congenital conditions, and other syndromes [1].

DDH's most common symptoms differ from one baby to another; the leg may appear shorter on the side of the dislocated hip, the leg on the side of the dislocated hip may turn outward, the folds in the skin of the thigh or buttocks may appear uneven, or the space between the legs may look wider than normal [3]. The symptoms of DDH may resemble other medical conditions of the hip. Thereby, a baby's physician must always be consulted for a diagnosis. DDH can be diagnosed using X-ray or Ultrasound imaging. Specific treatment for DDH is determined by the baby's physician; treatment options may include nonsurgical positioning device or placement of a pavlik harness [4], casting or surgery. However, these methods are either invasive or discomfort and not automatic [1], [5]. To this purpose, our aim is to develop a novel smart hip abduction splint (Smart HAS) device, in order to allow the physician to control automatically and accurately the degree of treatment of the device.

The paper is organized as follows. Section I provides the literature review and the objectives of the innovative study. Section II presents the materials and prototype of the Smart HAS. Section III showcases the simulated and experimental results of the Smart HAS. Section IV provides the discussion and the statistical evaluation of the Smart HAS as opposed to pavlik harness and conclusion, and section $\mathrm{V}$ provides the future work. 


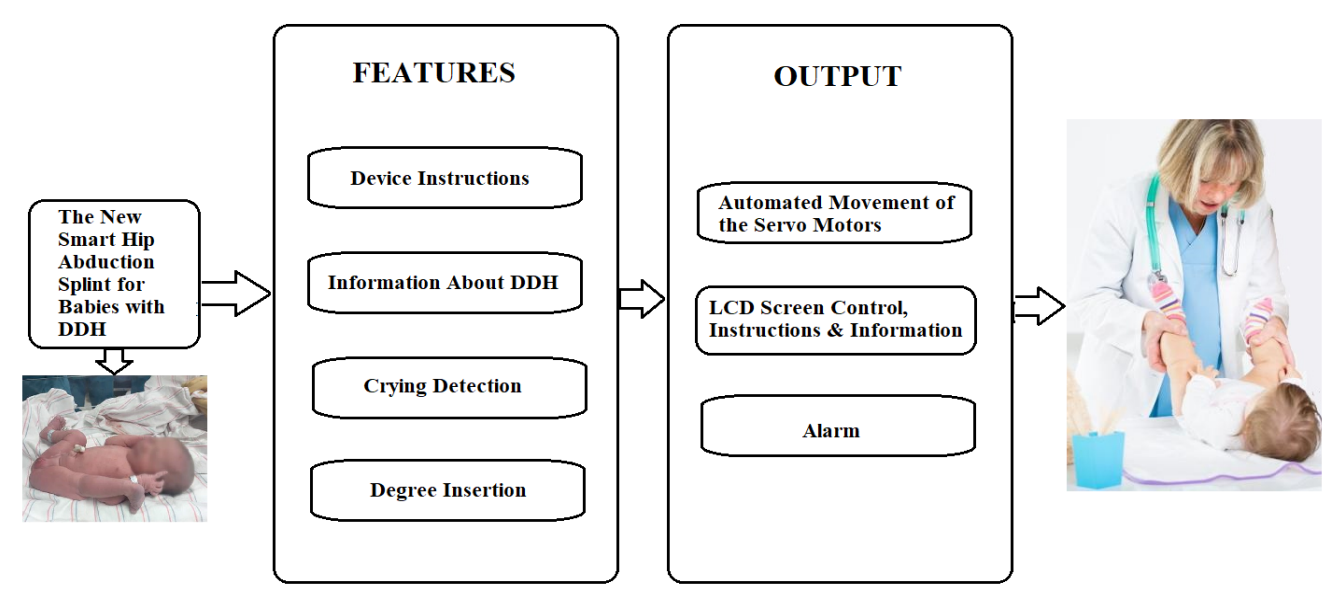

Fig. 1: The Prototype's Block Diagram of the Smart Hip Abduction Splint.

\section{MATERiALS}

The materials used to develop our Smart HAS are Nextion touchscreen [6], that is used to control the degree of the device by controlling two servomotors, the Blink application which allows to control the degree of opening of the device but from a distance, the Plexiglas and plastic parts and the Arduino Mega [7] for programming motors.

\section{Methodology and System Design}

Several steps were used to build our Smart HAS design. The steps were summarized in the block diagram shown in Fig. 1, from the simulated prototype features to the end user of the Smart HAS. The block diagram shows that after diagnosing the baby with DDH, the physician can insert a precise angle for treatment. The device was programmed to provide both the instructions on how to use the device and crucial information about the disease on the touchscreen. Then, a sensor for detecting the cry sound was attached to the car seat to activate the alarm in our device and alert the parents. The servomotors were controlled automatically by the angle inserted on the touchscreen to provide an adequate treatment angle. Then Arduino Mega was used for programming the main part of the device and controlling other parts. To move the Plexiglas and the plastic to the required degree of opening as inserted by the user, two servo motors were employed. The information were inserted using Nextion HMI 2.4" LCD touch screen and a Blink application that was rendered especially for this device, controlled the degree of opening of the servomotors. Using the Arduino Bluetooth module, the information inserted in the application allowed the servomotors to move as required.

To recognize the voice of the baby's cry with its exact frequencies, the microphone amplifier module was used. The sound sensor was used to detect any sound from the surrounding and produce an output signal. Thereby, any sound that was able to produce a vibration for the membrane of the microphone was detected, and an output signal in the form of electrical current was formed. However, in our system we needed only to sense the sound of the cry of the baby, and to program an output as a response to this cry. Thus, the input audio was analyzed, and a response was programmed only if the sound corresponded to the baby's cry, otherwise nothing occurred.

In order to program our system to function only when the baby's cry sound was detected, we have studied the characteristics of the signal of the baby's cry empirically. We studied the nature of this signal, monitored its rate of repetition, and determined its frequency. The sound of the baby's cry had a frequency that ranges between $700 \mathrm{~Hz}$ and $900 \mathrm{~Hz}$. Thereby, our system was programmed in a way to allow the detection of the sound of the baby's cry only.

Moreover, an alarm notification was sent to the user, meanwhile a music was played by the Arduino Mega and the speaker, inserted in the device, to calm down the baby for five minutes. Besides, the Plexiglas and the plastic used were covered by sponge and fabric for providing safety and comfort to the baby.

\section{Simulation Results}

The AutoCAD simulation are used to draw and plan for the geometrical aspects of the device that were going to implement, the system is formed of the Nextion Screen that is inside the Plexiglas box which is attached to the Plexi bars that holds the two servomotors, attaching to them at the terminals the plastic coated with the sponges and fabric to hold the baby's legs. The dimensions needed for the screen with a side length was $15 \mathrm{~cm}$ and the servomotors place with side length $10 \mathrm{~cm}$. The side bars which are attached to the legs of the baby with a height $18.5 \mathrm{~cm}$. Fig. 2 represents the AutoCAD dimensions and views of the whole system, i.e. reflecting the realistic shape of the system. Noteworthy that the colors that were chosen for the system were the black, red and white colors.

\section{EXPERIMENTAL RESULTS}

The compact design of the Smart HAS is shown in Fig. 3, this design provided a comfortable and flexible movement of the phantom and thus the baby. In addition, the user interface pages are shown in Fig. 4. On the start-up page the user was capable of choosing to know more about the disease, to have 


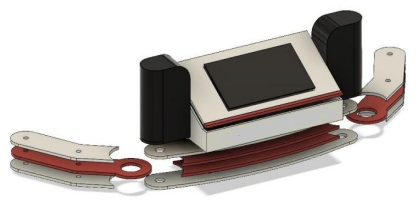

(a)

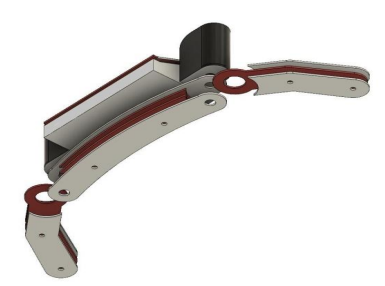

(c)

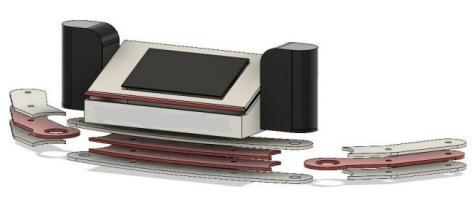

(b)

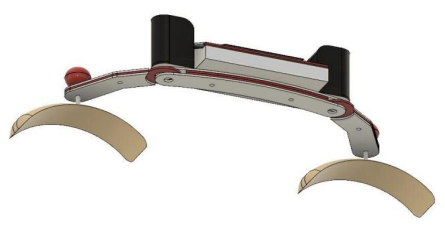

(d)

Fig. 2: The Autocad Simulation Results of the Smart HAS. (a) The Left Side View of the Parts and the Touchscreen. (b) The Right Side View. (c) The Bottom View. (d) The Overall View of the Simulated Smart HAS.

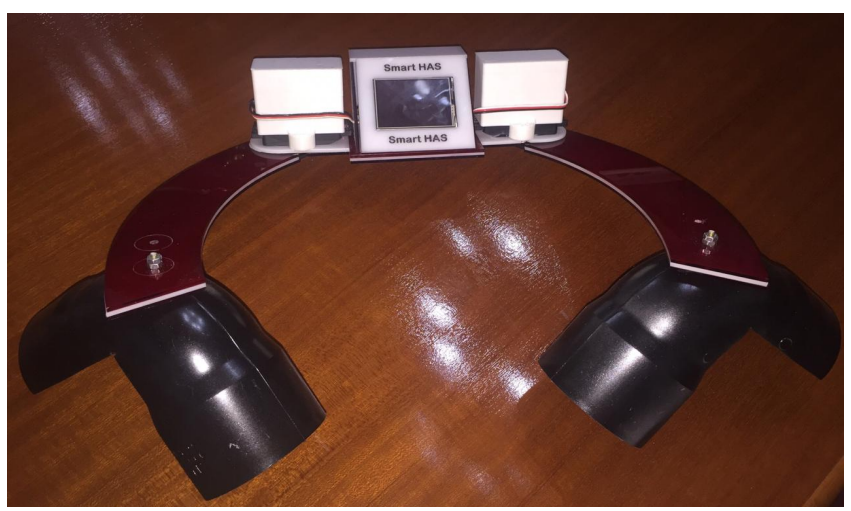

Fig. 3: Smart Hip Abduction Splint (HAS) for Developmental Dysplasia.

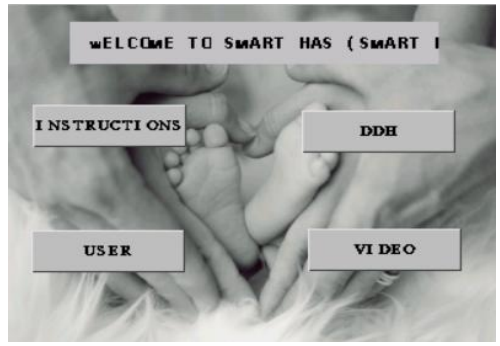

(a)

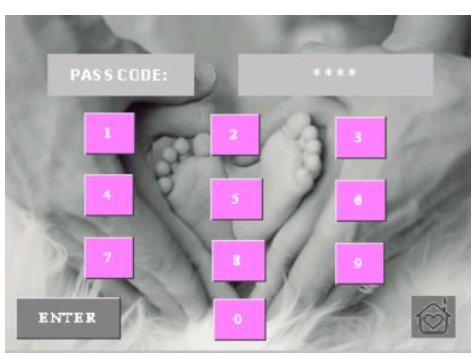

(b)

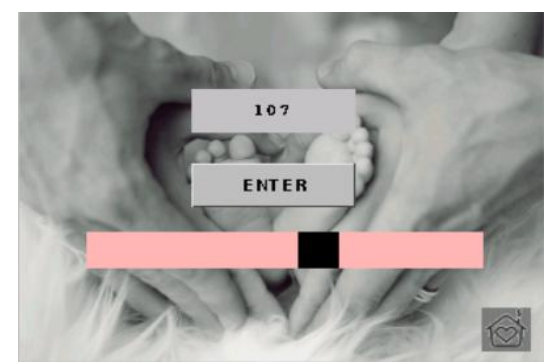

(c)

Fig. 4: User Interface and Touchscreen Results. (a) The Welcoming Page on the Touchscreen. (b) The Login Page Used by the Physician on the Touchscreen. (c) The Page Allowing to Input the Angles on the Touchscreen. 


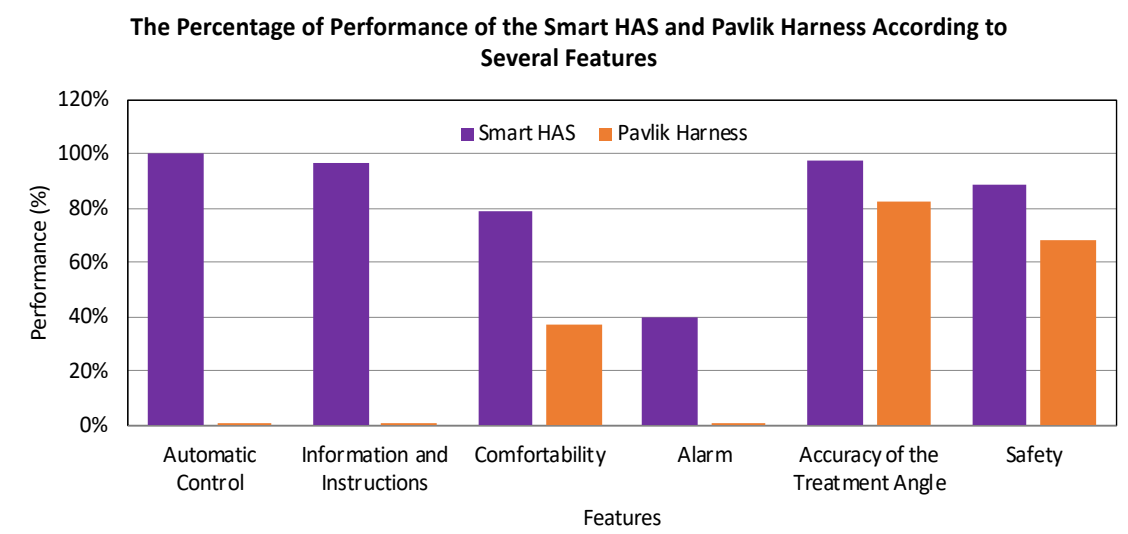

Fig. 5: The Statistical Evaluation in Percentage (\%) of Our Smart HAS with respect to the Pavlik Harness According to the Automatic Control, Information and Instructions Provided, Comfortability, Alarm, Accuracy of the Treatment Angle and Safety.

instructions or to insert the angle of treatment (if the user was the physician). Results also showed that the physician was able to insert the password to control the opening angle automatically. If the user input a wrong password it remains on the same page, but if the password is correct, the system moves to the page permitting the selection of the angle of treatment, where the physician can insert the angle and consequently the motors move as programmed. Moreover, the statistical evaluation, in percentage (\%), of our Smart HAS with respect to the pavlik harness were provided in in Fig. 5. These two design were evaluated according to the Automatic Control, Information and Instructions Provided, Comfortability, Alarm, Accuracy of the Treatment Angle and Safety.

\section{DISCUSSION AND CONCLUSION}

The statistical evaluation shown in Fig. 5 was the result of surveies carried out in some hospitals in Lebanon. These statistics were used to show the percentage of improvement of our Smart HAS compared to the old pavlik harness, by reporting the features that they provided. Features like automatic control, information and instructions and alarms were absent in the old device (0\%) [4], but they were present in higher percentages in our new device $40 \%$ and $97 \%$, respectively as reported in Table I. Comfortability, accuracy of the opening angle, and safety were higher in Smart HAS than in pavlik harness; which are very important features in the treatment process. The accuracy of performance of our Smart HAS was $98 \%$. i.e. $15 \%$ higher than that of the pavlik harness.

The improved pavlik harness, which we called "Smart Hip Abduction Splint", was showcased in details in both the simulation and experimental steps. All the materials needed to achieve the overall functionality of the Smart HAS were provided. Based on the simulations, experimental results and statistics our innovative device was worthwhile the development compared to the existing pavlik harness device.
TABLE I: The Maximum and Minimum Percentages (\%) of Performances of Both the Smart HAS and the Pavlik Harness Based on the Statistical Evaluation.

\begin{tabular}{|l|l|l|l|l|l|l|}
\hline & $\begin{array}{l}\text { Automatic } \\
\text { Control }\end{array}$ & $\begin{array}{l}\text { Information } \\
\text { and In- } \\
\text { structions }\end{array}$ & $\begin{array}{l}\text { Comforta- } \\
\text { bility }\end{array}$ & Alarm & $\begin{array}{l}\text { Accuracy } \\
\text { of the } \\
\text { Treat- } \\
\text { ment } \\
\text { Angle }\end{array}$ & Safety \\
\hline $\begin{array}{l}\text { Smart } \\
\text { HAS }\end{array}$ & $\mathbf{1 0 0 \%}$ & $97 \%$ & $79 \%$ & $40 \%$ & $\mathbf{9 8 \%}$ & $89 \%$ \\
\hline $\begin{array}{l}\text { Pavlik } \\
\text { Har- } \\
\text { ness }\end{array}$ & $0 \%$ & $0 \%$ & $37 \%$ & $0 \%$ & $83 \%$ & $68 \%$ \\
\hline
\end{tabular}

\section{FUTURE WORK}

For further improvement, various sensors that ensure the maximum safety and monitor the baby's condition can be added. Moreover, as the Smart HAS communication was achieved at a distance using bluetooth, thereby we could further add an application that works on WiFi so the physician can control the degree from a distance.

\section{REFERENCES}

[1] Orthobullets, "Developmental dysplasia of the hip (ddh) - pediatrics orthobulle," [online], May 2019.

[2] Orthoinfo, "Developmental dislocation (dysplasia) of the hip (ddh)," [online], May 2019.

[3] Stanfordchildrens, "Stanford children's health," [online], May 2019.

[4] J. Ruiz, J. Helwig, G. Espinoza, K. Sverrisdottir, and S. Jones, "An experimental verification of computational models demonstrating hip reductions via pavlik harness in developmental dysplasia of the hip," 2016.

[5] A. Martin, "Developmental dysplasia of the hip (ddh)-review of major imaging techniques." European Congress of Radiology 2017, 2017.

[6] N. Display, "2.4" nextion nx3224t024 hmi tft intelligent lcd touch display," [online], May 2019.

[7] Robotdyn, "Mega 2560 pro (embed) ch340g/atmega2560-16au," [online], May 2019. 